\title{
Effect of potassium doping on electrical properties of carbon nanotube fibers
}

\author{
M. Salvato, M. Lucci, I. Ottaviani, and M. Cirillo* \\ Dipartimento di Fisica and MINAS Laboratory, Università di Roma Tor Vergata, I-00133 Roma, Italy \\ E. Tamburri, I. Cianchetta, V. Guglielmotti, S. Orlanducci, and M. L. Terranova \\ Dipartimento di Scienze e Tecnologie Chimiche and MINAS Laboratory, Università di Roma Tor Vergata, I-00133 Roma, Italy \\ M. Pasquali \\ Chemical and Biomolecular Engineering, MS-362, Rice University, Houston, Texas 77251-1892, USA \\ (Received 3 October 2011; revised manuscript received 29 November 2011; published 12 December 2011)
}

\begin{abstract}
We investigate the effect of potassium $(\mathrm{K})$ doping on the transport properties of aligned single-walled carbon nanotube fibers. The temperature dependence of the electrical resistance, the current-voltage characteristics, and the magnetoresistance vs external magnetic field of the fibers consistently show that doping enhances the metallic character of the fibers and that the response of the samples can be quantitatively explained in two thermal regimes separated by a characteristic temperature $T^{*}$. At temperatures higher than $T^{*}$, the data are interpreted in the framework of variable range hopping theory, suggesting that the increased conductance with potassium doping is due to the increase of the density of states, which enhances carriers hopping. For temperatures below $T^{*}$, experimental evidence of fluctuation temperature-induced tunneling mechanism suggests that the doping by $\mathrm{K}$ atoms affects the potential barriers established between adjacent carbon nanotubes, enhancing the metallic properties of the fibers.
\end{abstract}

DOI: 10.1103/PhysRevB.84.233406

PACS number(s): 73.63.Fg, 61.46.Fg, 73.22.-f, 78.67.Ch

Carbon nanotubes (CNTs) are promising materials in electronics due to the potential wide range of applicability, ${ }^{1}$ but one difficulty foreseen for large-scale developments is the fabrication of samples having homogeneous electrical properties. Aggregates, mats, or fibers are commonly formed by CNTs having both semiconducting and metallic properties, a peculiarity which limits the spectrum of possible applications. ${ }^{2}$ At present, several techniques are under investigation in order to overcome this problem, and one specific technique consists in doping the CNT aggregate in order to increase the charge carriers density and favoring a semiconducting-to-metallic transition of the whole aggregate. ${ }^{3}$ Metallic doping by using halogen ${ }^{4}$ and alkali atoms ${ }^{5}$ has been successfully investigated. In this Brief Report, we show that doping a single-walled CNT (SWCNT) fiber with metallic potassium atoms improves its electrical conductance, as reported for other types of CNT aggregates. ${ }^{3,6,7}$ Moreover, interpreting the experimental data on the basis of the current theories for disordered noncrystalline materials, we provide a quantitative model for the role played by $\mathrm{K}$ atoms on the electrical properties of CNT fibers.

The fibers studied here have an external diameter of $100 \mu \mathrm{m}$ and consist of aligned CNTs having a diameter of $1 \mathrm{~nm} .{ }^{8}$ In order to generate the $\mathrm{K}$ doping, discrete fiber pieces are loaded in a small vial ( $\sim 20 \mathrm{ml}$ volume) and loaded uncapped into a bigger flask $(150 \mathrm{ml})$ capped after the addition of $\sim 1 \mathrm{~g}$ of solid potassium. The enclosed system is baked up to $120^{\circ} \mathrm{C}$ to melt the potassium and saturate the flask with $\mathrm{K}$ vapors, and the fibers are kept in contact with the $\mathrm{K}$ vapors for $2 \mathrm{~h}$. The presence of $\mathrm{K}$ inside the fibers is confirmed by energy dispersive spectroscopy (EDS), which detected a change of about $13 \%$ going from the surface to the center of the fiber, while the average value of the $\mathrm{K}$ concentration is $33 \%$. An electron microscope image of the fiber surface is shown in the upper inset of Fig. 1 together with the section used for inner and outer EDS analysis after K doping (lower inset).

The low-temperature measurements were performed in liquid $\mathrm{He}$ bath or vapor with the fibers connected (in a fourprobe scheme) to gold pads patterned on a silicon substrate, which in turn was secured to a copper sample holder housing a thermometer. Temperatures below $4.2 \mathrm{~K}$ were reached by pumping on He bath using auxiliary mechanical and diffusive pumps, and magnetotransport measurements were performed by using a 6-T superconducting magnet. The synthesized fibers were several meters long, and from these, we obtained samples a few centimeters in length that we used for the experiments. The results herein presented were typical for dozens of samples that we investigated.

Figure 1 shows data of the resistance of the fiber plotted vs $1 / T^{1 / 4}$, a measurement performed using $10 \mu \mathrm{A}$ as bias current. Such a value of the bias current generates a rather low power dissipation ${ }^{9}$ which ensures that no self heating (thermal) effects are present during the measurements. The decrease of the resistance for the doped fiber (FK) sample with respect to the bare fiber $(F)$ is evident in the whole temperature range investigated. In the plot of Fig. 1, we see that the data are very well fitted by the expression ${ }^{10} R / R_{0}=e^{\left(T_{M} / T\right)^{1 / 4}}$ where $T_{M}=18.2 / k_{B} N\left(\varepsilon_{F}\right) \xi^{3}$ is the Mott temperature, $k_{B}$ the Boltzmann constant, $N\left(\epsilon_{F}\right)$ the density of states at the Fermi level, and $\xi$ the localization length. A linear dependence down to a temperature $T^{*} \cong 2.8 \mathrm{~K}$ and $T^{*} \cong 4.0 \mathrm{~K}$ for $\mathrm{F}$ and $\mathrm{FK}$ systems, respectively, is observed. This evidence indicates, according to the variable range hopping (VRH) theory, that a three-dimensional (3D) hopping mechanism dominates the conduction process inside both the samples for $T>T^{*}$. The fit to the data gave $T_{M}=24700 \mathrm{~K}$ and $T_{M}=2000 \mathrm{~K}$ for $\mathrm{F}$ and FK, respectively. Any other $R$ vs $T$ dependence based on VRH, i.e. 1D and 2D VRH, would not provide linear dependencies shown in Fig. 1. 


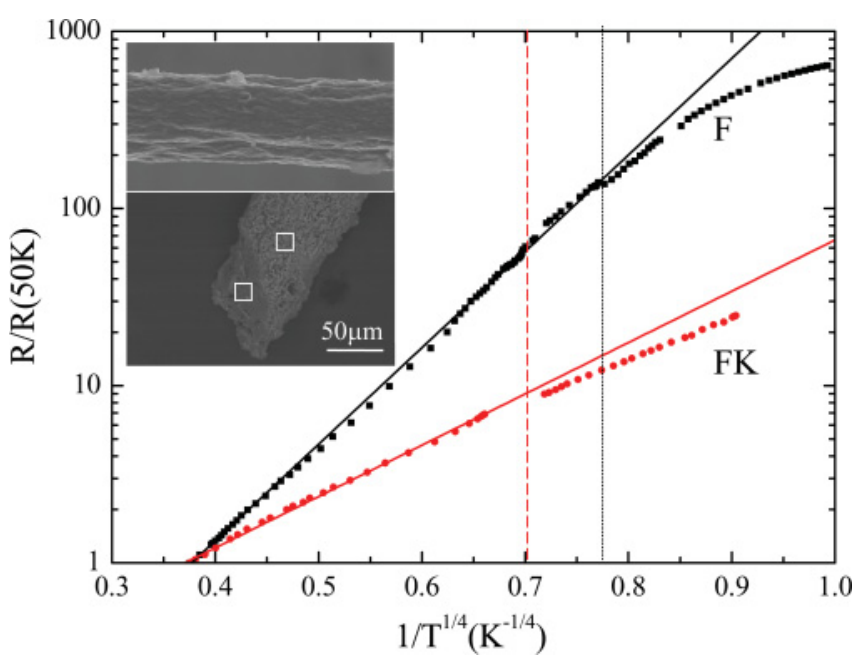

FIG. 1. (Color online) Normalized resistance vs $1 / T^{1 / 4}$ for fiber (F) and K-doped fiber (FK). The lines are fit to data above $T^{*}$, following 3D VRH. Insets: representative scanning electron microscope images of one of the fibers before doping (upper) and its section after doping (lower). The white squares represent approximately the area where the EDS probe was localized.

In order to better characterize the role played by $\mathrm{K}$ atoms inside the fiber, we performed magnetoresistance $(M R)$ measurements by probing the electrical resistance of the samples biased with the same current $10 \mu \mathrm{A}$ in an external magnetic induction $B$ going up to $5 \mathrm{~T}$ perpendicular to the fiber axis. The variation of the resistance with respect to its value at zero field is shown in Fig. 2 for the two types of samples. The negative value of $M R$ is expected in 3D localized transport ${ }^{11}$ and is usually interpreted as a competition between the shrinking of the electron wave functions at low fields, which enhances the resistance, and the interference between electron wave functions, which gives an opposite contribution, decreasing the $M R .^{12}$ Adding the two effects, the $M R$ data can be represented in this framework by the expression ${ }^{13}$ $M R=-a B+b B^{2}$, where the parameter $a=c_{\text {sat }} / B_{\text {sat }}$ takes into account the importance of the shrinkage effects, and the parameter $b \cong t_{1}\left(e^{2} a_{0}^{4} / \hbar^{2}\right)\left(T_{M} / T\right)^{3 / 4}$ accounts for the

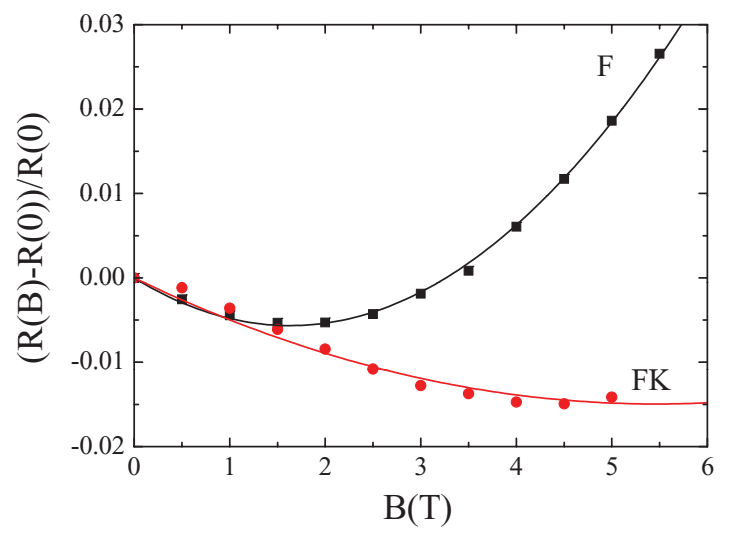

FIG. 2. (Color online) Magnetoresistance vs external magnetic field for $\mathrm{F}$ and FK. The lines are fits to the data according with parabolic behavior in localized systems. relevance of the interference effects. In these expressions, $c_{\text {sat }}$ is a constant, $B_{\text {sat }}=3 h /\left(e a_{0}^{2}\right)\left(T T_{M}\right)^{3 / 8}, t_{1}=0.00248, e$ is the electron charge, and $a_{0} \cong \xi .{ }^{14}$ This model is valid in the limit of low fields $B<B_{c}=\left(6 \hbar / e a_{0}^{2}\right)\left(T_{M} / T\right)^{-1 / 4}$. Both samples show negative $M R$ as a proof of the localized transport mechanism. Nevertheless, the parabolic behavior is much more evident in the case of the bare $\mathrm{F}$ fibers, while the metallic contribution of the $\mathrm{K}$ doping manifests itself in the saturation tendency of the $M R$ at high fields.

The curves in Fig. 2 are the fits to the data obtained using $a$ and $b$ as fitting parameters in the $M R$ vs $B$ expression. In particular, the values of $b$, together with the values of $T_{M}$ obtained by the fits of Fig. 1, allowed evaluating the localization lengths $a_{0}$, which result to be 4.7 and $5.3 \mathrm{~nm}$ for F and FK, respectively. Also the $B_{c}$ field limit is calculated by substituting inside its expression the obtained $a_{0}$ and $T_{M}$ values and obtaining $B_{c}$ higher than $19 \mathrm{~T}$ for both the samples, which corroborates the hypothesis of applicability in the range of our data $\left(B<B_{c}\right)$.

The values of $T_{M}$ and $a_{0}$ obtained by independent measurements enable us to interpret the role played by the presence of $\mathrm{K}$ in doped CNT fibers. Substitution of these parameters inside the $T_{M}$ expression allows calculating the density of states at the Fermi level $N\left(\epsilon_{F}\right)$ for the two systems. The calculation gives $N\left(\epsilon_{F}\right)=1.8 \mathrm{eV}^{-1} \mathrm{~nm}^{-1}$ for $\mathrm{F}$ samples, in very good agreement with the result found by other authors on similar systems. ${ }^{7}$ The same calculation operated in the case of FK samples gives $N\left(\epsilon_{F}\right)=20.2 \mathrm{eV}^{-1} \mathrm{~nm}^{-1}$. The obtained values of $N\left(\epsilon_{F}\right)$ suggest that the increased conductivity after $\mathrm{K}$ doping can be ascribed to the increase in the density of states (roughly one order of magnitude). The electrons, moving by hopping between localized states, find more available paths in the FK rather than in the $\mathrm{F}$ system because of the increased number of available states. In this view, the hopping lengths given by ${ }^{14} r=0.38\left(T_{M} / T\right)^{1 / 4} \xi$ results shorter in the case of FK $(9.4 \mathrm{~nm})$ with respect to $\mathrm{F}(15.6 \mathrm{~nm})$, as calculated at $T=$ $4.2 \mathrm{~K}$, according to the higher density of states present in the former system.

We also performed measurements of $I-V$ characteristics, which helped us to gain insight into the transport mechanism and to explain the behavior of the electrical resistance below $T^{*}$; in this range, as shown in Fig. 1 , its variations with temperature do not follow a $T^{-1 / 4}$ dependence. Figures 3(a) and 3(b) show the $I-V$ characteristics at different temperatures for $\mathrm{F}$ and FK samples, respectively. Both the systems show an ohmic behavior at high temperature, whereas a nonlinear shape is present at low temperature. In particular, the nonlinearity is more evident for F-type samples [Fig. 3(a)], evidencing a more pronounced semiconducting nature. At low temperature, the shapes of these curves are typical of the systems where the transport mechanism is affected by the presence of potential barriers. This statement is further strengthened by the observation that, as shown in the inset of Fig. 4, at low temperature the $R$ vs $T$ data are well fitted by the expression $R / R_{0}=e^{T_{1} /\left(T+T_{0}\right)}$ obtained by the fluctuation induced tunneling (FIT) ${ }^{15}$ model; this model assumes that the charge carriers move across potential barriers that, in the case of CNT aggregates, can be identified as the junctions between neighboring CNTs. ${ }^{16}$ The same model also gives for $I-V$ dependence the expression $I=I_{0} e^{-T_{1} /\left(T+T_{0}\right) \cdot(V / A-1)^{2}}$, where $T_{1}$ and $T_{0}$ are the same 

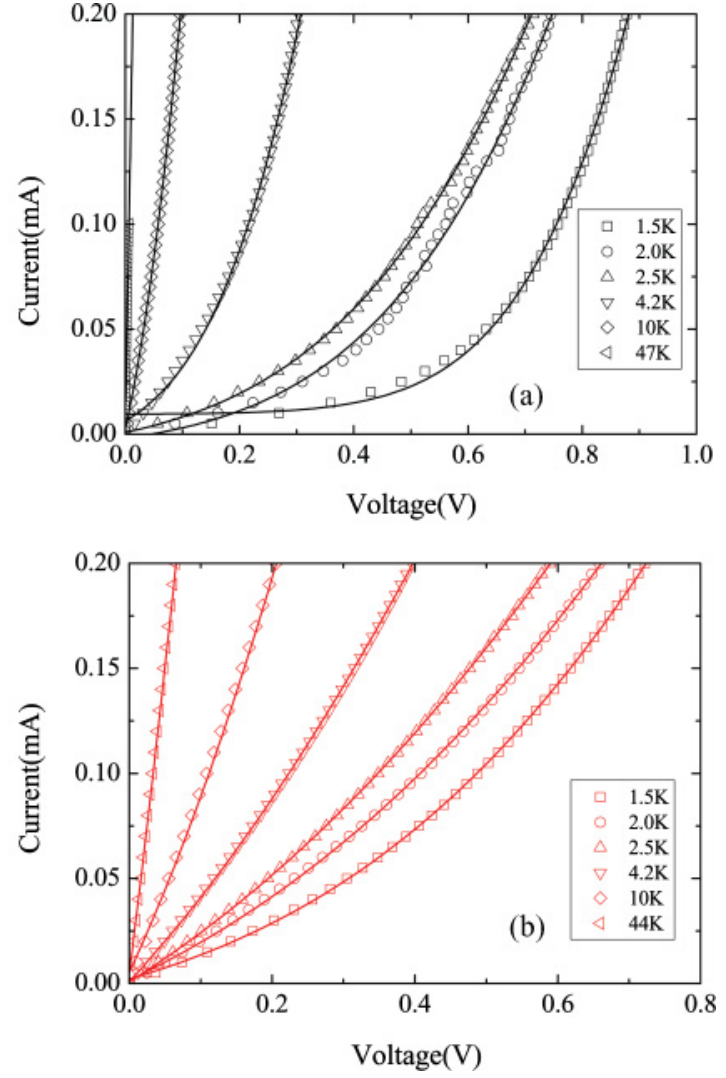

FIG. 3. (Color online) Current voltage characteristics at different temperature for (a) F and (b) FK samples. The lines are fits to the data following the FIT model.

parameters present in the $R$ vs $T$ expression, and $I_{0}$ and $A$ are further normalization parameters. ${ }^{15}$ In the FIT expressions, the two parameters $T_{1}$ and $T_{0}$ contain the barrier potential height $V_{0}$, the width $w$, and the contact area. The curves in Fig. 3 are fit to the data using the FIT expression for $I-V$ dependence. The ratio $T_{1} / T_{0}=\pi w \sqrt{2 m^{*} V_{0}} / 2 \hbar$ does not depend on the contact area, and its determination allows estimating the $w \sqrt{V_{0}}$ product. Here, $m^{*}=7.8 \times 10^{-32} \mathrm{~kg}$ is the effective electron mass for the CNT and $\hbar$ is the Planck constant divided by $2 \pi$. The data reported in Fig. 4 show the temperature dependence of this product obtained by fitting the $I-V$ curves for both the samples following the FIT model. ${ }^{15}$ Since it seems reasonable to consider $w$ temperature independent, the data in

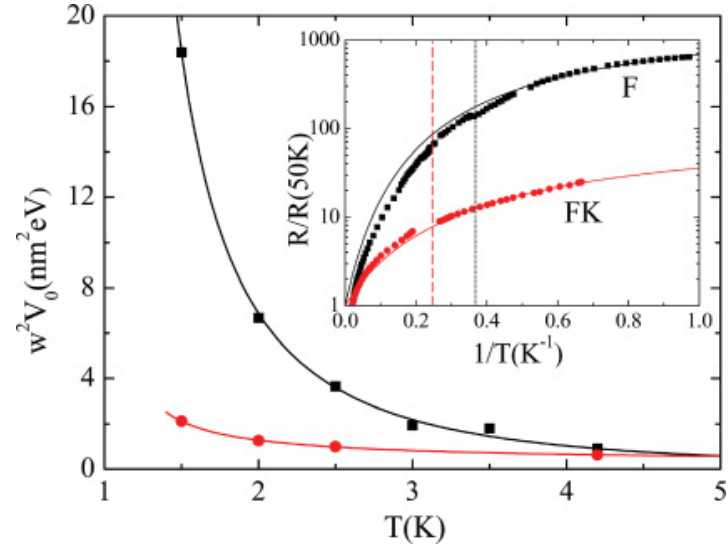

FIG. 4. (Color online) Temperature dependence of the product between potential barrier height $V_{0}$ and width $w$ as obtained by the data fittings of Fig. 3. Squares and circles refer to $F$ and FK, respectively. Inset: same data shown in Fig. 1 but plotted as $1 / T$. The lines are fit to the data below $T^{*}$ following FIT model.

Fig. 4 represent the temperature dependence of the potential barrier $V_{0}$. As expected, $V_{0}$ decreases with the temperature and becomes constant for $T>4 \mathrm{~K}$, where VRH instead of the FIT model works as confirmed by $R$ vs $T$ measurements. An estimate of $V_{0}$ can be obtained for both the samples substituting the localization length $a_{0}$ for $w$ in the data of Fig. 4. This gives $V_{0}=734 \mathrm{meV}$ and $V_{0}=85 \mathrm{meV}$ at $T=1.5 \mathrm{~K}$ for $\mathrm{F}$ and FK systems, respectively. The lowest value found at all temperature in the case of the doped fiber confirms the effect of $\mathrm{K}$ atoms to improve the electrical conductivity also at low temperature and the hypothesis of a tunneling mechanism, which takes place through a lower potential barrier with respect to the case of undoped CNTs.

In conclusion, we have investigated quantitatively the effect of $\mathrm{K}$ doping fibers of aligned CNTs; we have found that the doping reduces the electrical resistance according to two mechanisms which take place in two different temperature ranges. Above a given temperature $T^{*}$, the potassium doping causes an increase in the density of states and a consequent increase of the hopping probability of the charge carriers between localized states. Below $T^{*}$, a tunneling mechanism is responsible for charge transport, and the $\mathrm{K}$ doping lowers the potential barriers height with respect to the values measured in the case of the bare fibers. "cirillo@roma2.infn.it

${ }^{1}$ P. M. Ajayan and Otto Z. Zhou, in Carbon Nanotubes Synthesis, Structure, Properties and Applications, edited by S. M. Dresselhaus, G. Dresselhaus, and Ph. Avouris (Springer, Berlin, 2001), p. 391.

${ }^{2}$ A. B. Kaiser, Rep. Prog. Phys. 64, 1 (2001).

${ }^{3}$ R. S. Lee, H. J. Kim, J. E. Fischer, A. Thess, and R. E. Smalley, Nature 388, 255 (1997).

${ }^{4}$ L. Grigorian, K. A. Williams, S. Fang, G. U. Sumanasekera, A. L. Loper, E. C. Dickey, S. J. Pennycook, and P. C. Eklund, Phys. Rev. Lett. 80, 5560 (1998); N. Bendiab, R. Almairac, S. Rols, R. Aznar, J. L. Sauvajol, and I. Mirebeau, Phys. Rev. B 69, 195415 (2004);
M. Baxendale, V. Z. Mordkovich, S. Yoshimura, R. P. H. Chang, and A. G. M. Jansen, ibid. 57, 15629 (1998).

${ }^{5}$ X. Liu, T. Pichler, M. Knupfer, and J. Fink, Phys. Rev. B 70, 245435 (2004); G. Maurin, F. Henn, B. Simon, J. F. Colomer, and J. B. Nagy, Nano Lett. 1, 75 (2001); M. Bockrath, J. Hone, A. Zettl, P. L. McEuen, A. G. Rinzler, and R. E. Smalley, Phys. Rev. B 61, R10606 (2000).

${ }^{6}$ R. S. Lee, H. J. Kim, J. E. Fischer, J. Lefebvre, M. Radosavljevic, J. Hone, and A. T. Johnson, Phys. Rev. B 61, 4526 (2000);

L. Grigorian, G. U. Sumanasekera, A. L. Loper, S. Fang, J. L. Allen, and P. C. Eklund, ibid. 58, R4195 (1998). 
${ }^{7}$ J. Vavro, J. M. Kikkawa, and J. E. Fischer, Phys. Rev. B 71, 155410 (2005).

${ }^{8}$ L. M. Ericson, H. Fan, H. Peng, V. A. Davis, W. Zhou, J. Sulpizio, Y. Wang, R. Booker, J. Vavro, C. Guthy, A. N. G. Parra-Vasquez, M. J. Kim, S. Ramesh, R. K. Saini, C. Kittrell, G. Lavin, H. Schmidt, W. W. Adams, W. E. Billups, M. Pasquali, W. F. Hwang, R. H. Hauge, J. E. Fischer, and R. E. Smalley, Science 305, 1447 (2004). ${ }^{9}$ E. Pop, D. A. Mann, K. E. Goodson, and H. Dai, J. Appl. Phys. 101, 093710 (2007).

${ }^{10}$ N. F. Mott and E. A. Davis, Electronic Processes in Non-Crystalline Materials (Clarendon, Oxford, 1979), Chap. 2, p. 35.
${ }^{11}$ A. Kawabata, Solid State Commun. 34, 431 (1980).

${ }^{12}$ M. Benzaquen, D. Walsh, and K. Mazuruk, Phys. Rev. B 38, 10933 (1988).

${ }^{13}$ M. Jaiswal, W. Wang, K. A. Shiral Fernando, Y. P. Sun, and R. Menon, Phys. Rev. B 76, 113401 (2007).

${ }^{14}$ R. Rosenbaum, T. Murphy, E. Palm, S. Hannahs, and B. Brandt, Phys. Rev B 63, 094426 (2001).

${ }^{15}$ P. Sheng, Phys. Rev. B 21, 2180 (1980).

${ }^{16}$ M. Salvato, M. Cirillo, M. Lucci, S. Orlanducci, I. Ottaviani, M. L. Terranova, and F. Toschi, Phys. Rev. Lett. 101, 246804 (2008). 